\title{
Consistent Processing and Measurement Procedure on Micro Cutting
}

\author{
Tetsuya NARUSE ${ }^{1}$, Kazu ANDOU ${ }^{2}$, Yutaka KAMEYAMA², Masayoshi MIZUTANI², \\ Yoshihiro UEHARA ${ }^{3}$, Kazutoshi KATAHIRA', Weimin LIN $^{4}$, Chikako SASAKI ${ }^{2}$, Hitoshi OHMORI ${ }^{2}$, \\ ${ }^{1}$ Materials Fabrication Laboratory, RIKEN (The Institute of Physical and Chemical Research), \\ 1-7-13 Kaga Itabashi-ku, Tokyo, 173-0003, Japan, \\ ${ }^{2}$ Materials Fabrication Laboratory, RIKEN (The Institute of Physical and Chemical Research), \\ 2-1 Hirosawa, Wako, Saitama, 351-0198, Japan, \\ ${ }^{3} \mathrm{~V}$-CAD Fabrication team, RIKEN (The Institute of Physical and Chemical Research), \\ 1-7-13 Kaga Itabashi-ku, Tokyo, 173-0003, Japan, \\ ${ }^{4} \mathrm{~V}$-CAD Fabrication team, RIKEN (The Institute of Physical and Chemical Research), \\ 2-1 Hirosawa, Wako, Saitama, 351-0198, Japan,
}

\begin{abstract}
:
Miniaturization and weight reduction of portable audiovisual equipments, palmtop computers and mobile phones is being urgently pursued, increasing the demand for miniaturized and highly functional electronic devices, optical devices and mechanical components. A demand for high dimensional accuracy in parts comprising these products is also on the rise. Therefore, tool and processing using it are asked for high accuracy. In this research, we developed the system by which production of micro-tool and processing of work-piece are consistent. It carries an ELID grinding system and measurement equipment on a plane in precision 4-axis processing machine. It became production of a tool with detailed high accuracy is possible, and the deflection of a tool can be suppressed to $30 \mathrm{~nm}$.
\end{abstract}

Keywords: Micro cutting, Desk-top machine tools, Micro tool, Micro mechanical fabrication

\section{Introduction}

Miniaturization and weight reduction of portable audiovisual equipments, palmtop computers and mobile phones is being urgently pursued, increasing the demand for miniaturized and highly functional electronic devices, optical devices and mechanical components. A demand for high dimensional accuracy in parts comprising these products is also on the rise. Micro-tools having an outer diameter, at the tip, of less than several tens of microns play a leading role in "cutting-edge" R\&D and in the development of new industrial technologies. For example, they support a wide variety of "cutting-edge" needs, such as the micro-fabrication of various tools used for semiconductor devices, microlens arrays, measurement microprobes, and bio-manipulators. In a micro-fabrication, micro-tools used for micro-punching and micro-cutting require sufficient mechanical strength to withstand the load during machining. In order to produce a high precision tool, it is required to repeat machining and measurement. However, if a tool is removed from the machine, accuracy will fall. Moreover, the setup for using a micro tool for processing is very difficult. Therefore, it may be made to damage when a micro tool is used for processing. Moreover, the sharpness of the edge part of a tool also has big influence on the surface roughness of a processing side.

Today, Japanese industry has been faced to lose many middle-aged technicians because of their retirements. Therefore, the setup which needs high-level skill is very difficult. We are developing the "processing template" system $[1,2]$ as part of a NEDO project[3]. It supports passing young laborers technology. Then, we developed the system for production and processing of a tool carried grinding system and on-machine measurement device in precision 4-axis machine. In this research, in order to produce a high precision micro tool, feedback processing was performed on the processing machine. I think that the deflection under tool rotation can be reduced by carrying out tool production on a processing machine. The effect of the deflection and sharpness of tool exerted on the processing surface in a consistent on-machine measurement system was investigated.

\section{Development of advanced consistent on-machine measurement system}

Figure 1 shows the exterior of the machine developed specifically for micro-tool machining. The machine is designed to be extremely compact, and can be used on a desktop [4]. This machine has three linear axes, X, Y, and Z. Also, an indexing unit can be mounted as another rotational axis for workpieces to be fabricated not only into cylindrical shapes, but also, if desired, into columnar shapes of square, elliptical cross-sections or pyramidal shapes having corners. In order to improve the shape accuracy of desired micro-tools, an image analysis type profilometer (resolution: 0.1 $\mu \mathrm{m}$ ) was installed on the machine. 
On-machine measurement device is a non-contact image pick-up system, and combines a high resolution camera system and an image processing system. Measurement of a tool diameter and deflection are possible for this device. Moreover, it can be used also for the tool tip position detection at the time of machining. [5] Thus, feedback processing from an initial diameter of several hundreds of micrometers to final diameter of several micrometers, has become possible without removal of the micro-tools from the machine for measurement. Feedback processing was performed from the tool which diameter of $200 \mu \mathrm{m}$ to $5 \mu \mathrm{m}$. The results between target size and size error at micro punching are shown in Fig.2. Processing of submicron accuracy is realized by feeding back measurement data. In addition, the material which I used is hard metal.

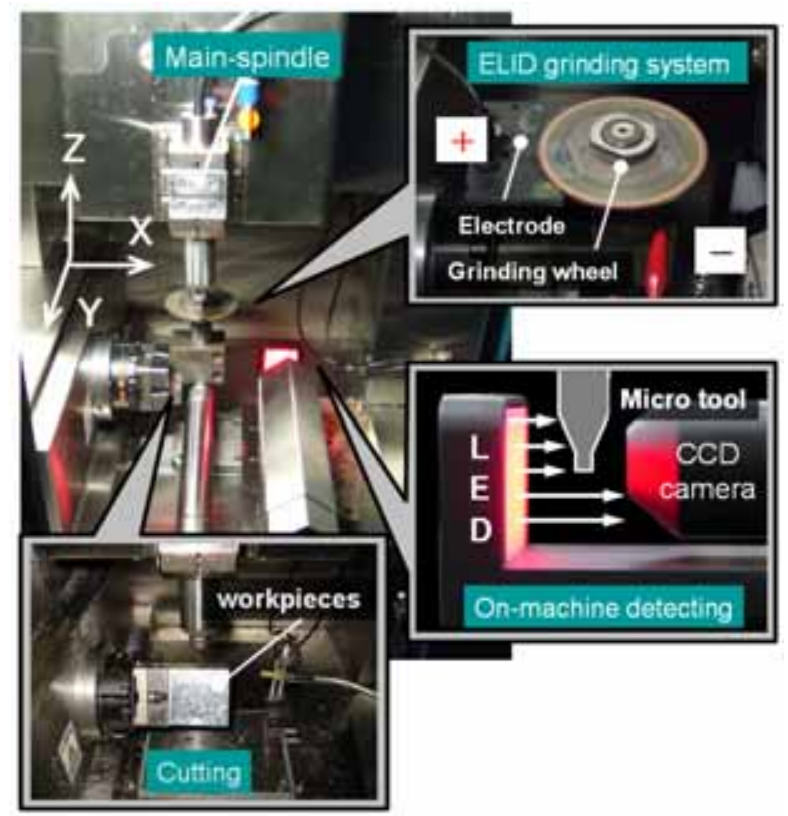

Fig.1 Advanced consistent on-machine measurement system.

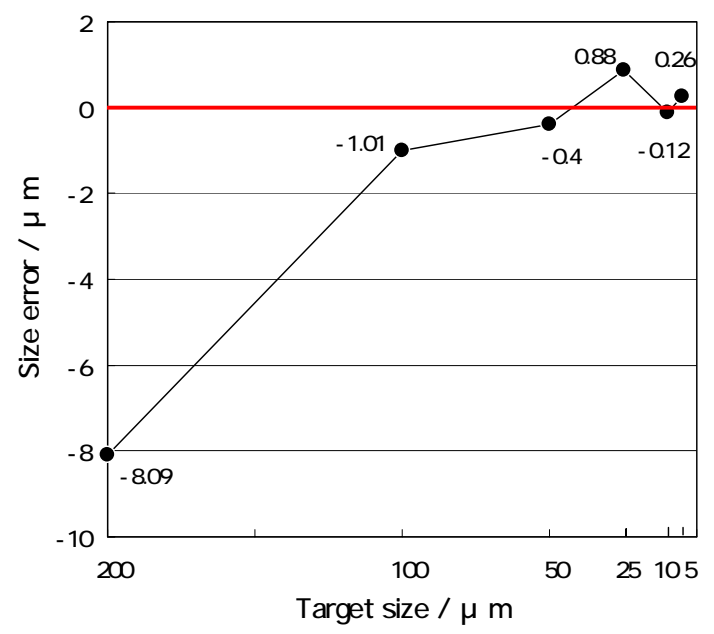

Fig.2 Measurement size to a target size.
If chucking of the tool is carried out, deflection will certainly arise. It depends on the accuracy of a tool or a chuck. An expert skill is necessary for those adjustments. In detailed cutting, deflection has big influence on the processing surface or a tool. The result of having measured deflection in on-machine measurement device is shown in Fig.3. The deflection in the usual chucking is about $6 \mu \mathrm{m}$. A tool may be damaged when it is processed in this state using a tool of about $10 \mu \mathrm{m}$ in diameter. Compared with it, the deflection of on-machine produced micro tool is $0.03 \mu \mathrm{m}$. Deflection of a micro tool can be made very small if a tool is produced in the developed consistent type system. It is because the portion which was swaying at the time of chucking was deleted. Processing conditions could be improved in order not to add excessive power to a tool in processing. Moreover, improvement in processing accuracy is expectable. By detecting the tip position of a tool by image processing, the junction point with workpieces will become exact (Fig.4).

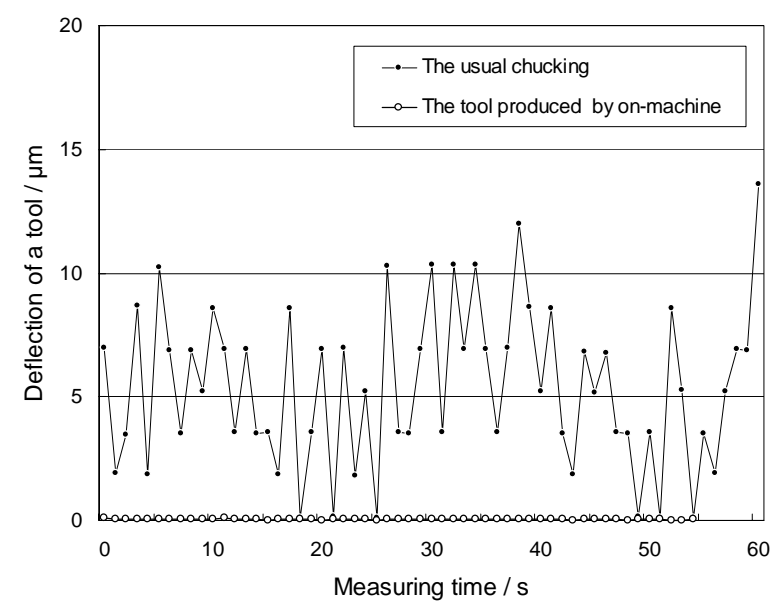

Fig.3 Deflection measurement result by on-machine measurement device.

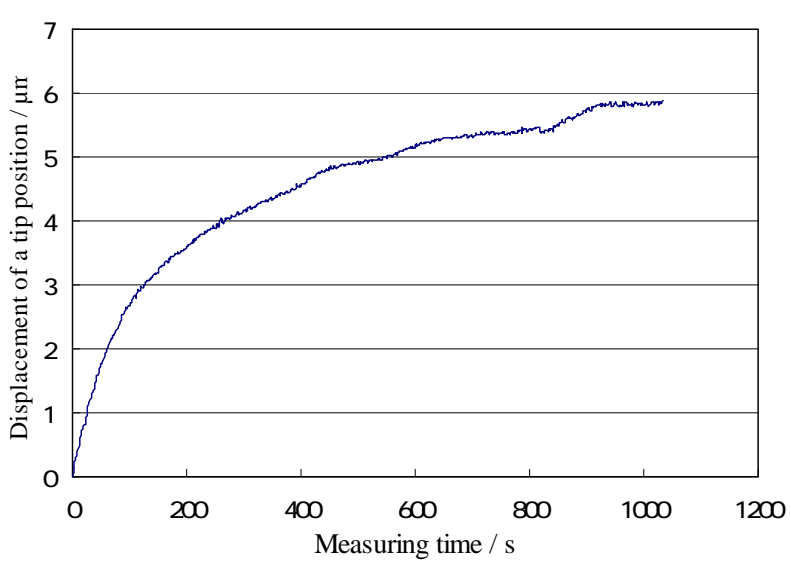

Fig.4 Displacement measurement of the tool tip position. 


\section{Machining characteristic in consistent processing system}

Micro-end-mill was produced in order to investigate the influence of the deflection exerted on detailed processing. The tool is a one-sheet edge and diameter is $100 \mu \mathrm{m}$. The whetstones used for processing are \#325 and \#20000. The SEM observation photograph of a micro-end-mill is shown in Fig. 5. The surface roughness of a tool improves and by using grinding wheel of \#20000 shows that edge is also sharper. The slot processing experiment was conducted on the tool in the case where tool exchange is performed, and after tool production on a plane. SUS316 was used for the work material. Processing conditions are shown in Table 1. The SEM observation photograph of a slot processing result is shown in Figs.6 and 7. Figure 6 shows the microphotograph of a bottom part. The big difference to each sample is not seen. It is because the effect of deflection is small in a bottom part. However, the processed surface is wavy when there is deflection in a side part, as shown in Fig. 7. In this case, accuracy and surface roughness worsen. Moreover, this phenomenon may give damage to a tool. Damage may be given to a tool. On the other hand, wave is not seen in a case without deflection. That surface roughness is constant and shows good surface quality.

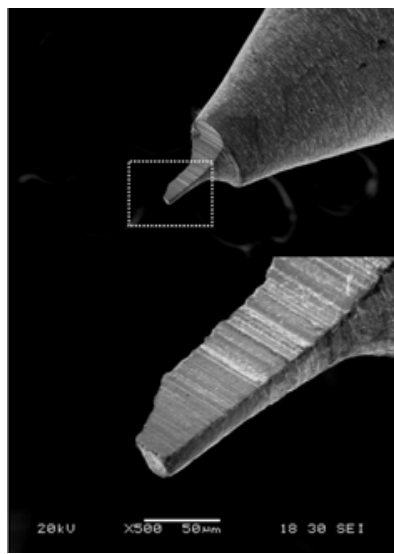

(a) \#325

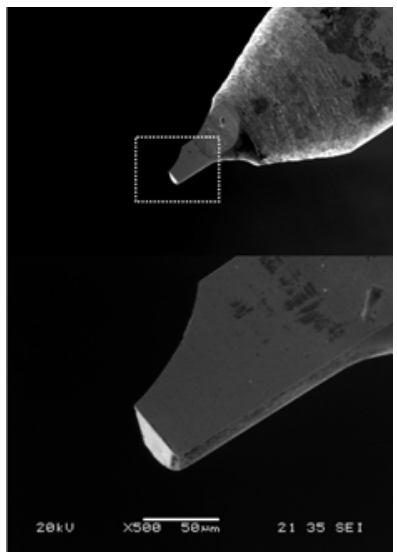

(b) \#20000
Fig.5 SEM observation of micro-end-mill.

Table1 1. Cutting condition

\begin{tabular}{|c|c|}
\hline Tool rotational speed & 40000(min-1) \\
\hline Total depth of cut & $100 \mu \mathrm{m}$ \\
\hline Depth of cut & $2 \mu \mathrm{m} /$ pass \\
\hline Feed speed & $10 \mathrm{~mm} / \mathrm{min}$ \\
\hline Processing length $(\mathrm{mm})$ & $5 \mathrm{~mm} / \mathrm{line}$ \\
\hline Work material & SUS316 \\
\hline Coolant & Kerosene \\
\hline
\end{tabular}

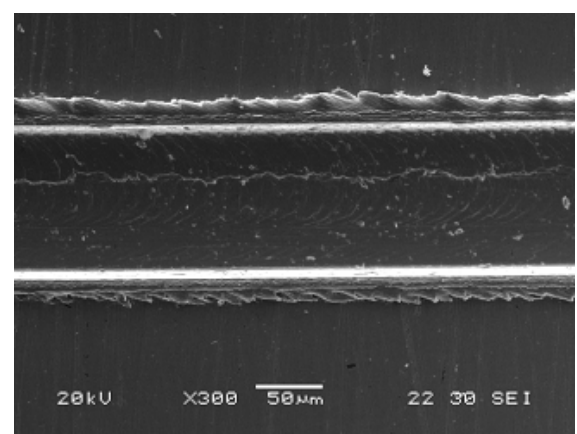

(a) When there is any tool deflection.

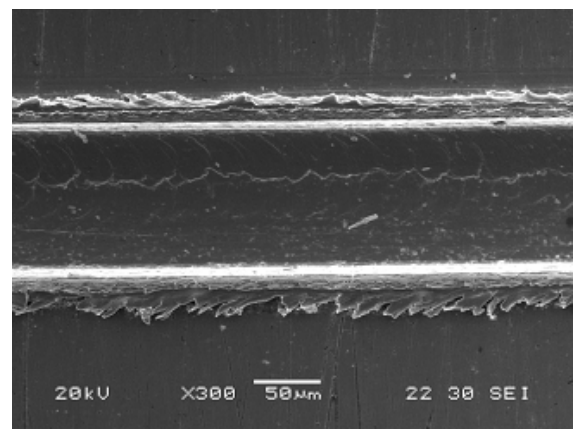

(b) When there is no tool deflection.

Fig.6 Microphotograph at the bottom part of machined workpieces.

(The used tool was produced by the grinding wheel of \#325.)

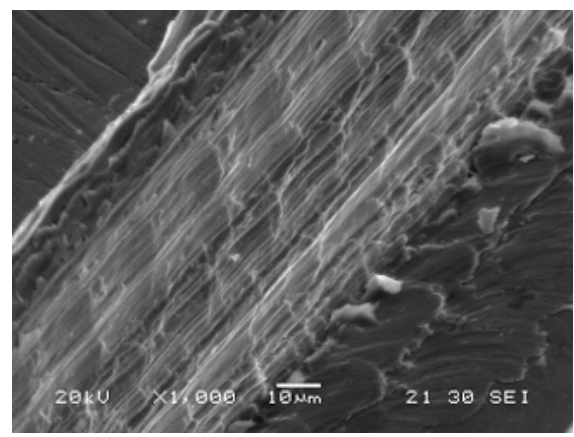

(a) When there is any tool deflection.

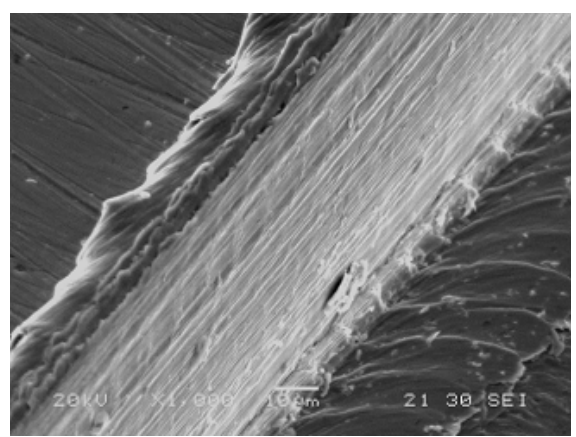

(b) When there is no tool deflection.

Fig.7 Microphotograph at the side part of machined workpieces.

(The used tool was produced by the grinding wheel of \#325.)

The milling result by the tool produced by grinding wheel of \#20000 is shown in Fig. 8. The edge of tool produced by the 
grinding wheel of \#20000 is sharper than grinding wheel of \#325. Therefore, the surface roughness is better. This factor is so important that a tool becomes small. Moreover, Fig. 9 shows the result of measuring wear of tool tip $\mathrm{R}$ using on-machine measurement device. Tip $\mathrm{R}$ of tool was became $14 \mu \mathrm{m}$ when it was processed $25 \mathrm{~mm}$ in a case with deflection. Compared with it, wear of tool tip $\mathrm{R}$ is small when there is no deflection. From these results, by reducing deflection of tool, surface quality of machined field was able to improve and wear of tool was able to be controlled.

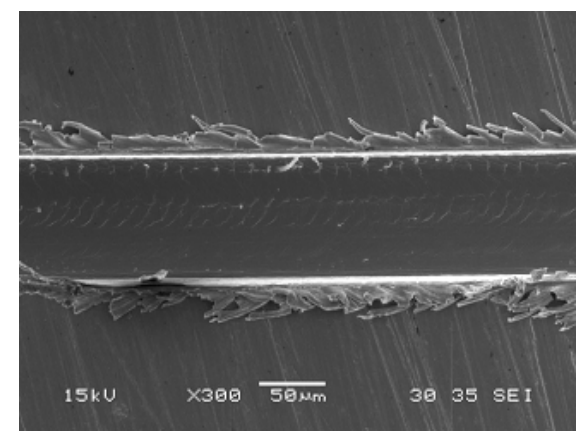

(a) Microphotograph of bottom part.

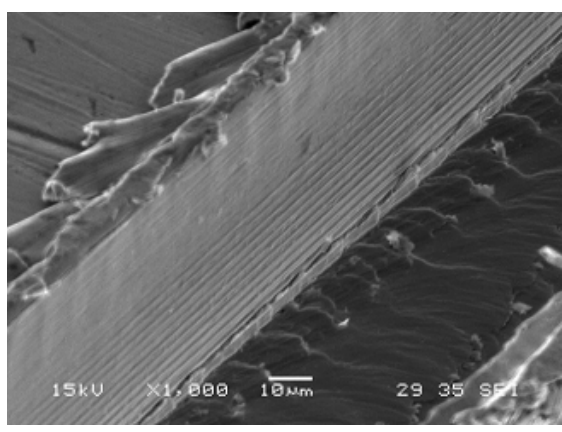

(b) Microphotograph of side part.

Fig.8 Microphotograph of machined workpieces.

(The used tool was produced by the grinding wheel of \#20000.)

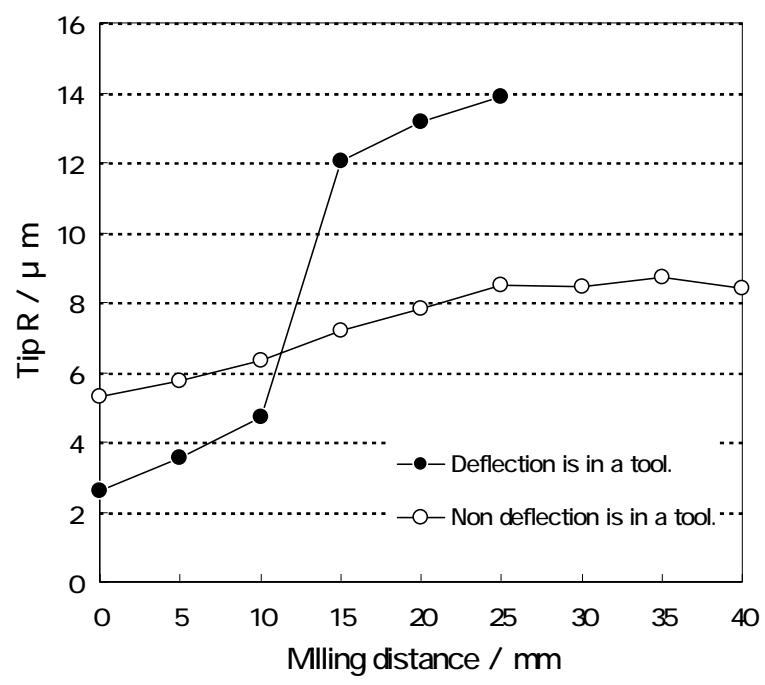

Fig.9 Relation between processing distance and wear of tool.

\section{Conclusions}

In the present study, the advanced consistent on-machine measurement system was developed, and the predominancy of this system was verified. As a result, the high precision micro tool was producible by advanced consistent on-machine measurement system. Moreover, deflection of the tool was able to be made very small when rotating a spindle. Surface roughness was able to be controlled by reducing deflection. Probably it will be space-saving that these characteristics have been satisfied in desk-top machine tools, and it will be effective also in a cost down.

\section{References}

[1] T. Naruse, K. Andou, M. Mizutani, Y. Kameyama, H. Ohmori, M. Tsuneki, 2007, Die and Mould Technology, Construction of the Processing-Template to Support the Succession of Techniquesof, Vol.22, No.8, pp.152-153

[2] K. Andou, T. Naruse, Y. Kameyama, M. Mizutani, Y. Koma, H. Ohmori, 2007, Die and Mould Technology, Systematization of Processing Knowledge for Construction of Process Templates, Vol.22, No.8, pp.154-155

[3] Web site: NEDO homepage http://www.nedo.go.jp/activities/portal/gaiyou/p06021/p0 6021.html

[4] H. Ohmori, Y. Uehara, K. Katahira, Y. Watanabe, T. Suzuki, W. Lin, N. Mitsuishi, 2005, Advanced Desktop Manufacturing System for Micro-Mechanical Fabrication, Laser Metrology and Machine Performance VII, pp.16-29.

[5] H. Hasegawa, 2006, JSAT, Dynamic measurement technology of a tool position, pp. 641-644. 